\title{
Análise da variabilidade da frequência cardíaca em pessoas vivendo com HIV submetidos à terapia antirretroviral (TARV) após a prática de atividade física
}

\author{
Analysis of heart rate variability in people living with HIV undergoing antiretroviral therapy \\ (TARV) after practicing physical activity
}

Análisis de la variabilidad de la frecuencia cardíaca en personas que viven con el VIH que reciben terapia antirretroviral (TAR) después de la actividad física

\author{
Tayná Oliveira de Gois \\ ORCID: https://orcid.org/0000-0001-9643-9453 \\ Universidade Tiradentes, Brasil \\ E-mail: taayna.oliveira@gmail.com \\ Cristiane Kelly Aquino dos Santos \\ ORCID: https://orcid.org/0000-0002-0260-7194 \\ Universidade Tiradentes, Brasil \\ E-mail: ckellyakin@gmail.com \\ Júlio Cesar Camargo Alves \\ ORCID: https://orcid.org/0000-0002-3169-7096 \\ Universidade do Estado de Minas Gerais, Brasil \\ E-mail: juliocamargo.edufisica@gmail.com \\ Estélio Henrique Martin Dantas \\ ORCID: https://orcid.org/0000-0003-0981-8020 \\ Universidade Tiradentes, Brasil \\ E-mail: estelio.henrique@ souunit.com.br
}

\begin{abstract}
Resumo
A Síndrome da Imunodeficiência Adquirida (AIDS) é uma doença causada pelo vírus da Imunodeficiência Humana (HIV) que ataca as células de defesa do indivíduo, os linfócitos TCD4+ causando um distúrbio grave do sistema imunológico. Seu tratamento é baseado em terapia combinada com medicações antirretrovirais que conseguem controlar a carga viral e diminuir as taxas de mortalidade da doença. Como alternativa, estratégias de intervenção através da prática de exercícios físicos com a finalidade de auxiliar no tratamento e qualidade de vida de pessoas vivendo com HIV vem sendo utilizadas pelos benefícios à saúde. Este estudo tem como objetivo comparar a variabilidade da frequência cardíaca (VFC) medida por um cardiofrequêncímetro, em pacientes HIV positivos submetidos a terapia antirretroviral (TARV) em repouso e após a realização de um treinamento concorrente (TC). O presente estudo constitui-se de uma pesquisa analítica, observacional e transversal. A amostra será composta por 15 pessoas que vivem com HIV e que se encontram em acompanhamento clínico ambulatorial do Serviço de Atendimento Especializado (SAE) (Casa Rosa) em Barretos interior do estado de São Paulo. Para apresentação dos dados foi utilizado o teste de Wilcoxon para encontrar quais foram diferentes, o nível de significância adotado foi de $\mathrm{p}<0,05$. Para verificar a relação dos resultados, foi utilizado o teste coeficiente de correlação de Spearman. O tamanho de efeito também foi calculado utilizando o teste de Cohen (d). Os dados analisados correlacionaram nível de significância em todas as variáveis no domínio da frequência e do tempo, excetuando SDNN e SD2. Houve tamanho de efeito grande somente para o parâmetro $\operatorname{HF}(\Delta \%=279,92 \%$ de redução) no domínio da frequência e nos parâmetros RMSSD $(\Delta \%=79,58 \%$ de redução) e SD1 ( $\Delta \%=79,62 \%$ de redução) no domínio do tempo no TC, nos outros parâmetros não houve efeito grande. Dessa forma, observa-se um que a retomada vagal, que normalmente ocorre após um treinamento físico, não ocorreu ou está retornando lentamente. Enquanto no parâmetro LF houve uma redução significativa, ou seja, uma retirada simpática após o TC, mesmo assim a retirada simpática não foi total, pois o parâmetro $\mathrm{LF} / \mathrm{HF}(\Delta \%=42,31 \%$ de aumento e $\mathrm{TE}=0,70)$, que seu aumento significa atividade simpática, mostrou um aumento significativo após o TC. Em conclusão a variabilidade da frequência cardíaca dos pacientes em estudo diminuiu após o treinamento concorrente possivelmente pela disfunção autonômica evidenciada na literatura para pessoas vivendo com HIV.
\end{abstract}

Palavras-chave: Exercício físico; Frequência cardíaca; Sorodiagnóstico da AIDS; Terapia Antirretroviral de Alta atividade. 


\begin{abstract}
Acquired Immunodeficiency Syndrome (AIDS) is a disease caused by the Human Immunodeficiency Virus (HIV) that attacks the individual's defense cells, the TCD4 + lymphocytes causing a serious immune system disorder. Its treatment is based on therapy combined with antiretroviral medications that manage to control viral load and decrease the mortality rates of the disease. As an alternative, intervention strategies through the practice of physical exercises in order to assist in the treatment and quality of life of people living with HIV have been used for health benefits. This study aims to compare the heart rate variability (HRV) measured by a cardiofrequency meter, in HIV positive patients undergoing antiretroviral therapy (ART) at rest and after performing concurrent training (CT). The present study consists of an analytical, observational and cross-sectional research. The sample will consist of 15 people living with HIV and who are undergoing outpatient clinical follow-up at the Specialized Care Service (SAE) (Casa Rosa) in Barretos, in the interior of the state of São Paulo. To present the data, the Wilcoxon test was used to find which ones were different, the level of significance adopted was $\mathrm{p}<0.05$. To verify the relationship of the results, Spearman's correlation coefficient test was used. The effect size was also calculated using the Cohen test (d). The analyzed data correlated the level of significance in all variables in the frequency and time domain, except for SDNN and SD2. There was a large effect size only for the parameter $\operatorname{HF}(\Delta \%=279.92 \%$ reduction $)$ in the frequency domain and in the parameters $\operatorname{RMSSD}(\Delta \%=79.58 \%$ reduction $)$ and SD1 $(\Delta \%=79.62 \%$ reduction $)$ in the time domain in the TC, in the other parameters there was no great effect. Thus, one observes that the vagal resumption, which normally occurs after physical training, has not occurred or is returning slowly. While in the LF parameter there was a significant reduction, that is, a sympathetic withdrawal after the CT, even so, the sympathetic withdrawal was not total, since the parameter $\mathrm{LF} / \mathrm{HF}(\Delta \%=42.31 \%$ increase and $\mathrm{TE}=0.70)$, that its increase means sympathetic activity, showed a significant increase after CT. In conclusion, the heart rate variability of the patients under study decreased after concurrent training, possibly due to the autonomic dysfunction shown in the literature for people living with HIV.
\end{abstract}

Keywords: Physical exercise; Heart rate; AIDS serodiagnosis; Highly active antiretroviral therapy.

\title{
Resumen
}

El Síndrome de Inmunodeficiencia Adquirida (SIDA) es una enfermedad causada por el Virus de Inmunodeficiencia Humana (VIH) que ataca las células de defensa del individuo, los linfocitos TCD4 + causando un trastorno grave del sistema inmunológico. Su tratamiento se basa en terapia combinada con medicamentos antirretrovirales que logran controlar la carga viral y disminuir las tasas de mortalidad de la enfermedad. Como alternativa, las estrategias de intervención a través de la práctica de ejercicios físicos para ayudar en el tratamiento y la calidad de vida de las personas que viven con el VIH se han utilizado para obtener beneficios para la salud. Este estudio tiene como objetivo comparar la variabilidad de la frecuencia cardíaca (VFC) medida con un medidor de cardiofrecuencia, en pacientes VIH positivos sometidos a terapia antirretroviral (TAR) en reposo y después de realizar entrenamiento concurrente (TC). El presente estudio consiste en una investigación analítica, observacional y transversal. La muestra estará compuesta por 15 personas que viven con el VIH y que se encuentran en seguimiento clínico ambulatorio en el Servicio de Atención Especializada (SAE) (Casa Rosa) en Barretos, en el interior del estado de São Paulo. Para presentar los datos se utilizó la prueba de Wilcoxon para encontrar cuáles eran diferentes, el nivel de significancia adoptado fue $\mathrm{p}<0.05$. Para verificar la relación de los resultados se utilizó la prueba del coeficiente de correlación de Spearman. El tamaño del efecto también se calculó mediante la prueba de Cohen (d). Los datos analizados correlacionaron el nivel de significancia en todas las variables en el dominio de la frecuencia y el tiempo, excepto para SDNN y SD2. Hubo un gran tamaño del efecto solo para el parámetro HF ( $\Delta \%=$ reducción del $279,92 \%)$ en el dominio de la frecuencia y en los parámetros $\operatorname{RMSSD}(\Delta \%=$ reducción del $79,58 \%)$ y $\operatorname{SD} 1(\Delta \%=$ reducción del $79,62 \%$ ) en el dominio del tiempo en el TC, en el resto de parámetros no hubo gran efecto. Así, se observa que la reanudación vagal, que normalmente ocurre después del entrenamiento físico, no se ha producido o está regresando lentamente. Mientras que en el parámetro LF hubo una reducción significativa, es decir, una retirada simpática tras el TC, aun así, la retirada simpática no fue total, ya que el parámetro LF / HF $(\Delta \%=42,31 \%$ de aumento y TE $=0,70)$, que su aumento significa actividad simpática, mostró un aumento significativo después de la TC. En conclusión, la variabilidad de la frecuencia cardíaca de los pacientes en estudio disminuyó después del entrenamiento concurrente, posiblemente debido a la disfunción autonómica mostrada en la literatura para personas que viven con el VIH.

Palabras clave: Ejercicio físico; Frecuencia cardiaca; Serodiagnóstico del SIDA; Terapia antirretroviral de gran actividad.

\section{Introdução}

O Vírus da Imunodeficiência Humana, Human Immunodeficiency Virus, (HIV), é um retrovírus com genoma RNA, da

Família Retroviridae e subfamília Lentivirinae, que através da transcriptase reversa consegue transcrever o RNA viral em uma cópia de DNA e integrar-se ao genoma humano. O vírus tem a capacidade de infectar as células de defesa - denominadas linfócitos, preferencialmente os TCD4+ -, aumentando a incidência de doenças oportunistas e consequentemente levando à Síndrome da Imunodeficiência Adquirida (AIDS). A Síndrome da Imunodeficiência Adquirida, Acquired Immunodeficiency 
Syndrome, (AIDS) é uma doença de estágio avançado caracterizada por deficiência do sistema imunológico do indivíduo infectado pelo HIV, resultando em imunossupressão progressiva. (Brasil, 2018; Silva, et al., 2018).

Desde a descoberta do HIV na década de 80 e as pesquisas relacionadas, principalmente após o surgimento do tratamento com os antirretrovirais, aumentaram as chances de sobrevivência se tornando uma doença crônica. A partir de 1996 o Brasil instituiu o tratamento gratuito dos pacientes com AIDS e em 2013 o governo passou a adotar para todos os estágios da doença independente da contagem de CD4. (Faria; Gude; Lima, 2018).

Até 2017 existiam cerca de 37 milhões de pessoas vivendo com HIV (PVHIV) em todo o mundo segundo o The Jointed United Nations Programme on HIV/AIDS (UNAIDS), e destes, 21,7 milhões faziam uso de terapia antirretroviral (TARV). No Brasil, até junho de 2018 foram notificados 982.129 casos de AIDS, dos quais 65,5\% eram do sexo masculino e cerca de $72 \%$ encontram-se em TARV. (Brasil, 2018)

O tratamento antirretroviral (TARV) estimulou a redução da morbidade e da mortalidade associadas à AIDS. Atualmente, mais de $90 \%$ das pessoas que fazem o tratamento completo apresentam resultados indetectáveis de carga viral e consequentemente com chances baixas de transmitir o HIV. Além da TARV, o governo também implementou a profilaxia pósexposição sexual ao HIV (PEP) em 2012 e a profilaxia pré-exposição (PrEP) sexual após dezembro de 2014 no SUS. (Pereira, et al., 2019)

Dentre os estados do Brasil, o Nordeste representa uma expansão da doença levando a uma situação de risco de infecção na população em geral. Apesar disso, qualquer pessoa está vulnerável, pois a transmissão é globalizada, dinâmica e instável dependendo somente do comportamento individual e coletivo. Observa-se uma forte associação com HIV e doenças sexualmente transmissíveis (DSTS) evidenciando a pouca utilização dos métodos de proteção para essas doenças, sendo necessária medidas educativas sociais. (Chaves, et al. 2014).

Apesar da melhora da sobrevida com a utilização da TARV, os efeitos colaterais também a acompanhou, como mudanças na distribuição da gordura corporal, resistência insulínica, alterações ósseas, aumento dos lipídios. (Pinto, et al., 2013). Além do envelhecimento natural, existe toda a carga de efeitos gerados pelo tratamento com os antirretrovirais mudando o padrão de mortes para causas não-HIV principalmente por doenças cardiovasculares. O exercício físico e a prática regular ajuda a manter um estilo de vida ativo e uma saúde metabólica favorável, diminuindo os fatores de risco cardiovasculares como obesidade, dislipidemia e hipertensão arterial. (Ozemek; Erlandson; Jankowski, 2020)

Pacientes infectados pelo HIV tem uma forte relação com disfunção autonômica, além do elevado risco cardiovascular. Ou seja, há um aumento na atividade nervosa simpática levando a uma redução do tônus vagal. Uma boa ferramenta para avaliar a função autonômica e risco de doença arterial coronariana desses pacientes é o uso da variabilidade da frequência cardíaca (VFC), por ser um método não invasivo e de fácil acesso. A VFC reflete a influência simpática e parassimpática sobre o nó sinusal através de variações observadas no eletrocardiograma nos intervalos de batimento a batimento (R-R). (Mcintosh, 2016)

Com a atuação do simpático há uma redução da VFC, já com a ação do parassimpático há um aumento da VFC. A interpretação se dá por meio da análise de dois domínios, tempo e frequência. Ela pode sofrer influência por condições como HIV, cardiomiopatia, idade avançada, comportamento emocional. (Mccraty; Shaffer, 2015).

Diante do exposto, já é sabido pelos profissionais de saúde que o exercício físico tem impacto positivo na saúde de forma geral: metabólica, imunológica, cardiovascular, entre outros. Isso envolve tanto pessoas vivendo com HIV quanto os HIV negativos, atuando então como uma ferramenta a mais no tratamento médico. Soma-se a isso a desregulação autonômica e o maior risco de eventos cardiovasculares presentes nas pessoas com HIV e em uso de TARV. Portanto, o objetivo deste estudo é avaliar variabilidade da frequência cardíaca em pessoas vivendo com HIV submetidos à terapia antirretroviral (TARV) em repouso e após a prática de um exercício físico. 
A TARV revolucionou o tratamento das pessoas vivendo com HIV, mas também trouxe preocupações relacionadas aos fatores de risco para doenças cardiovasculares, como hipertensão arterial sistêmica, hipercolesterolemia e hipertrigliceridemia, aterosclerose, disfunção endotelial, diabetes mellitus, entre outros. (Deeks; Lewin; Havlir, 2013)

Além disso, as infecções oportunistas e virais decorrentes das respostas imunes podem levar a uma cardiotoxicidade relacionada a medicações, imunossupressão a longo prazo, levando a uma deterioração da função cardiovascular. Isso tudo contribui com o desequilíbrio e disfunção autonômica e aumento de eventos cardiovasculares, como infarto do miocárdio, morte súbita, insuficiência cardíaca. Estudos baseados em meta-análises alertaram sobre o risco duas vezes maior de pessoas HIV+ desenvolverem doenças cardiovasculares. (Shah, et al., 2018)

Com o advento do tratamento com os antirretrovirais foi percebido uma diminuição da função do sistema nervoso simpático e parassimpático, aumentando o risco para DCV. Sendo assim, o tratamento com ART é considerado um fator de risco independente para DCV. É importante detectar fatores de risco controláveis para reduzir a chance de DCV em pacientes com HIV em TARV. (Godijk, et al. 2020)

A neuropatia autonômica desencadeada no HIV está associada ao aumento da morbidade desses pacientes, mesmo com a terapia antirretroviral combinada e supressão virológica, apresentam sintomas de disfunção do sistema autônomo. (Artal, 2017).

Em indivíduos sem disfunção autonômica o natural é que em repouso há predominância da modulação parassimpática cardíaca. Quando há uma desregulação, observa-se maior modulação simpática e menor modulação parassimpática cardíaca. (Dong, 2016).

A análise da VFC é um bom sinal na avaliação da função autonômica e do risco de doenças cardiovasculares. Uma alta VFC é interpretado como uma boa resposta e eficiência do sistema autonômico, enquanto que baixa VFC indica uma adaptação ineficaz do sistema nervoso autonômico. Essa disfunção autonômica identificada através da reduzida VFC já está presente mesmo nos estágios iniciais da infecção pelo vírus. (Quiles; Garber; Ciccolo, 2018; Artal, 2017).

Mesmo antes do surgimento da TARV, ela já era detectada em pacientes com HIV e AIDS, evidenciando um efeito neuropatológico do vírus. Dentre os sintomas da desregulação autonômica estão: disfunção secreto-motora e gastrointestinal, disfunção sexual nos homens, intolerância ortostática e alterações da pressão arterial. (Mcintosh, 2016; Chow, et al., 2015)

Além da disfunção autonômica pelo HIV poder ser por causa primária, estudos indicam também causas secundárias, tais como infecções desencadeada por citocinas, mecanismo do sistema imune e vasculopatia. Algumas pesquisas relacionam o uso de medicações, baseados na presença da neurotoxina, principalmente pela utilização da estavudina que leva a uma depleção mitocondrial neural e inibição de DNA polymerase. (Esiri, et al. 1993; Tyor, et al., 1992; Mittal et al., 2004; Lebech et al., 2007).

Esse desequilíbrio do sistema autônomo já no início da infecção pelo HIV pode ser também justificado pela preferência do vírus no sistema central, presente em altas concentrações no hipocampo, gânglios da base e outras regiões de regulação hipotalâmica. Possíveis alterações causadas pelo HIV no hipocampo poderiam justificar essa disfunção autonômica, já que o hipocampo é a região que comanda o sistema nervoso autônomo. (Chow, et al. 2011).

Estudos mostram a influência de emoções positivas com a VFC. Sentimentos como compaixão, apreciação, em oposição a sensações negativas como ansiedade e estresse, geram um ritmo cardíaco mais seguro. Essa coerência reflete em sensação de bem-estar e melhorias físicas, sociais e mentais. (Mccraty; Shaffer, 2015).

A VFC é baseada na variabilidade dos intervalos R-R do eletrocardiograma em dois domínios: do tempo (temporal) e da frequência (espectral). Pode ser de forma combinada ou exclusiva. A análise da VFC permite a diferenciação dos componentes simpáticos e parassimpáticos. Quando em alta frequência reflete atividade parassimpática, enquanto a VFC na 
faixa de baixa frequência é resultado do predomínio de atividade simpática. A razão dessas duas faixas de frequência cardíaca é um parâmetro de equilíbrio simpático-vagal. (Borges, Farinatti, 2011; Mittal, et al., 2004)

A VFC tem relação com o valor da FC, na medida em que a frequência aumenta, há menos tempo para que a variabilidade ocorra e a VFC diminui. Já quando a frequência diminui, há mais tempo entre os batimentos e a VFC aumenta. No entanto, pacientes idosos, HIV + e cardiopatas não há uma relação entre elas, não aumentando a VFC mesmo com reduções na FC. (Umetani, et al. 1996)

O domínio do tempo da VFC pode ser analisado pelos parâmetros média Normal-para-Normal (batimento-parabatimento) (NN) (intervalo RR), Desvio padrão dos intervalos NN (SDNN), intervalos de tempo entre ciclos adjacentes como raiz quadrada da somatória do quadrado das diferenças entre os intervalos RR (RMSSD), a raiz quadrada da somatória do quadrado das diferenças dos valores individuais em relação ao valor médio dividido pelo número de intervalos RR (RMSM), a porcentagem da diferença maior que 50 milissegundos entre intervalos RR adjacentes (pNN50), o Descritor padrão 1 (SD1) que é a variabilidade rápida de batimento-para-batimento nos intervalos RR e Descritor padrão 2 (SD2) que descreve a variabilidade a longo prazo. São considerados parâmetros gerais da VFC, ou seja, mostram tanto atividade simpática quanto parassimpática o SDNN e SD2, já o NN (principalmente em repouso), RMSSD, RMSM, pNN50 e SD1 representam a atividade parassimpática no domínio do tempo (Askgaard et al., 2011; Dong, 2016; Marães, 2010).

No domínio da frequência (rotação ou batimento por um período), temos como parâmetros a Alta frequência (HF) $0,15 \mathrm{~Hz}$ a $0,4 \mathrm{~Hz}$ ocorrendo em um período de 2,5 a 7 segundos, Baixa frequência (LF) 0,04 Hz a 0,15 Hz ocorrendo em um período de 7 a 25 segundos, Frequência muito baixa (VLF) $0,0033 \mathrm{~Hz}$ a $0,04 \mathrm{~Hz}$ ocorrendo em um período de 25 a 300 segundos, Ultra baixa frequência (ULF) valores menores que $0,0033 \mathrm{~Hz}$ ocorrendo em 333 segundos e Total Power. Assim como no domínio do tempo, no domínio da frequência podemos separar os parâmetros de acordo com o que representa na análise, como o HF representa a atividade parassimpática, o LF diferentemente do que alguns estudos mostram, não mede somente a atividade simpática, mede principalmente a atuação do baroreflexo, ou seja, pode medir tanto atividade simpática quanto parassimpática, VLF mede respostas de estresse pós-traumático, o ULF mede o ciclo cardíaco, respostas hormonais e metabólicas, e o Total Power fornece informações sobre como o poder é distribuído (a variação e amplitude de um dado ritmo) em função da frequência (o período de tempo de um determinado ritmo) (Mccraty; Shaffer, 2015). Um resumo dos parâmetros do domínio do tempo e da frequência da VFC está apresentado tabela a seguir:

Tabela 1. Resumo do domínio do tempo e da frequência na variabilidade da frequência cardíaca

\begin{tabular}{|c|c|c|c|}
\hline \multicolumn{4}{|c|}{ Variabilidade da frequência cardiaca (VFC) } \\
\hline \multicolumn{2}{|c|}{ Dominio do tempo } & \multicolumn{2}{c|}{ Dominio da frequência } \\
\hline Parassimpático & Simpático parassimmático & Parassimpático & Simpático/parassimpático \\
\hline NN & SDNN & HF & LF \\
\hline RMSSD & SD2 & & \\
\hline RMSM & & & \\
\hline pNN50 & & & \\
\hline SD1 & & & \\
\hline
\end{tabular}

Fonte: Autores.

Pessoas que vivem com HIV tem maiores chances de desenvolver doenças cardiovasculares devido a múltiplos fatores, dentre eles os diretamente relacionados ao vírus, pela dislipidemia consequente da TARV, inflamação, coagulação desregulada e monócitos ativados estão dentre os responsáveis. Esses fatores deterioram a função cardiovascular, esquelética e 
metabólica, prejudicando a aptidão cardiorrespiratória (cardiorespiratory fitness - CRF) uma medida prognóstica. São fatores que aumentam o CRF, a prática de exercício físico regular e o treinamento físico. (Ozemek; Erlandson; Jankowski, 2020)

A atividade física tem a capacidade de trazer melhorias na saúde ao longo da vida, como na regulação dos níveis de glicemia, função cardiovascular, hipertrofia muscular, controle da pressão arterial, equilíbrio dos níveis de colesterol, diminuição do estresse e qualidade de vida. Pessoas que vivem com HIV ativas comparadas as não ativas se beneficiam principalmente com a redução da lipodistrofia e obesidade. (Ozemek; Erlandson; Jankowski, 2020)

Comparada com populações HIV negativas, pessoas que vivem com HIV tem uma acentuada redução no estado funcional que é a capacidade de praticar atividades do dia a dia sozinhos para a sua necessidade básica. Além disso também apresentam baixos níveis de desempenho físico, somado ao fato de existir grande perda de massa, força muscular e densidade mineral óssea nessa população. A atividade física entra como uma nova terapia que beneficia a saúde mental, cardiovascular, pulmonar, osteomuscular, composição corporal, comorbidades associadas e qualidade de vida. A prescrição de exercício físico deve ser incluída no tratamento médico englobando uma equipe multidisciplinar. (Chetty; Cobbing; Chetty, 2020).

Com o advento da TARV complicações infecciosas como miocardite, foram reduzidas em compensação aumentou a prevalência de doenças arteriais periféricas e coronárias. Também há o envolvimento do tônus autonômico cardíaco relacionado a doença avançada por HIV. Foi observado disfunção autonômica global grave em pacientes vivendo com HIV sem associação com evidência clinica ou ecocardiográfica de doença cardíaca, sendo a AIDS o mecanismo da desregulação. (Costa; Almeida, 2015)

HIV soropositivos apresentam além dos fatores de risco tradicionais os envolvendo a doença como a infecção per se, duração da infecção, carga viral, terapêutica, resposta imunitária alterada. Orientações como dieta, perda de peso, cessação do tabagismo, exercício físico devem ser orientadas aos pacientes HIV positivos em uso de TARV. Além das alterações comportamentais, as terapêuticas também podem ser utilizadas para a redução do risco cardiovascular. (Costa; Almeida, 2015)

Em um estudo retrospectivo analisando as causas de morte em 13 cohorts HIV-1 na Europa e EUA, entre 1996-2006 em uso dos antirretrovirais (TARV), foi observado diminuição da mortalidade por causas diretamente relacionadas à doença e aumento por causas não relacionadas, como neoplasias, doença cardiovascular $(7,9 \%$ da mortalidade, sendo $40 \%$ por infarto do miocárdio/doença cardíaca isquêmica). Desta forma, observou-se que o processo de envelhecimento se tornará o principal fator na mortalidade por HIV na próxima década. (Gill, et al. 2010).

Uma análise de programa de prevenção com medidas não farmacológicas e farmacológicas quando necessário realizadas por Lima et al. Observaram alterações após 6 meses nos triglicerídeos, colesterol total e LDL e uma redução significativa nos indivíduos com alto risco cardiovascular. (Lima, et al. 2009)

\section{Metodologia}

Estudo transversal, observacional, ou seja, todos os voluntários (pessoas vivendo com HIV) participaram das intervenções agudas, aleatoriamente, sendo as variáveis independentes, uma sessão de TC, utilizando como variáveis dependentes pré e pós as respectivas intervenções, valores do domínio do tempo e da frequência da VFC.

Estudo transversal é um tipo de pesquisa que analisa a relação entre a frequência de uma doença ou condição interessada e outras variáveis da população em um determinado local e tempo. Ou seja, analisam a informação em um ponto no tempo ou em um intervalo de tempo pequeno. Ele nos permite conhecer a prevalência de fatores associados a uma doença através de um estudo simultâneo e relativamente rápido. Os estudos transversais constituem uma subcategoria dos estudos observacionais. No estudo observacional o pesquisador não intervém, apenas observa e registra as informações que lhe interessam para posterior análise. (Fronteira, 2013). 
O universo do presente estudo foi constituído por 611 indivíduos com sorologia positiva para HIV e que se encontram em acompanhamento clínico ambulatorial no Serviço de Atendimento Especializado (SAE) (Casa Rosa) em Barretos interior do estado de São Paulo.

O estudo foi divulgado por meio de palestras e cartazes entre agosto de 2015 e abril de 2016. As inscrições para participação foram realizadas no SAE, onde os voluntários a participar deixaram seu nome com a recepcionista para posterior contato do pesquisador por telefone para início do projeto. Após explanação dos objetivos do estudo, foi verificada sua predisposição para participação no estudo e avaliação dos critérios de inclusão e exclusão.

Critérios para inclusão:

Consentimento médico ou da equipe médica envolvida; Contagem de linfócito TCD4 acima de 250 células/mm3; Não possuir enfermidade oportunista no início da pesquisa; Carga viral indetectável; Foram aceitos indivíduos de ambos os sexos; Não ser praticante de treinamento físico regular há pelo menos seis meses, e ser maior de idade. Após a análise do prontuário (que consta de exames clínicos realizados periodicamente) dos participantes interessados, juntamente com seus respectivos médicos do SAE, foram incluídos no estudo aqueles que não apresentaram doenças cardiovasculares, ou qualquer outra, em que o risco de realizar exercício fosse maior do que o benefício. Quando os médicos e o pesquisador principal identificaram que o prontuário não estava claro, foram requisitados os exames clínicos ou cardiovasculares (foram necessários a realização de 2 eletrocardiogramas), para incluir o participante que estava apto a realizar exercícios moderados, com os riscos minimizados ao máximo.

Critérios de Exclusão:

Não apresentar consentimento médico ou da equipe médica envolvida; Contagem de linfócito TCD4 abaixo de 250 células/mm3; Possuir enfermidade oportunista no momento do início da pesquisa; Carga viral detectável; Menor de 18 anos; Praticante treinamento físico há pelo menos seis meses. Foram excluídos os participantes que após a análise do prontuário (que consta de exames clínicos realizados periodicamente), juntamente com seus respectivos médicos do SAE os que apresentaram patologias cardiovasculares ou qualquer outra em que o risco de realizar exercício fosse maior do que o benefício. Quando os médicos e o pesquisador principal identificaram que o prontuário não estava claro, foram requisitados os exames clínicos ou cardiovasculares, e após todos esses procedimentos quando os exames mostraram que não era seguro este participante realizar exercícios moderados, foi então excluído do estudo.

O $n$ amostral foi baseado em estudos científicos publicados e que são similares ao desse trabalho. Os artigos base utilizados como referencial tiveram 10 indivíduos soropositivos (Garcia et al., 2014), 12 indivíduos soronegativos (Matsunaga et al., 2008), 15 indivíduos soronegativos (Santiago et al., 2013), 13 indivíduos soropositivos e 10 indivíduos soronegativos (BORGES et al., 2012). Em nosso estudo, completaram todos os procedimentos 15 voluntários soropositivos.

O presente estudo atende às normas para a realização de pesquisa em seres humanos, de acordo com a Resolução 466/2012, do Conselho Nacional de Saúde de 12/12/2012, e foi aprovado pelo CEP da UNIRIO com o CAAE 45756115.5.0000.5285 e parecer: 1.180 .516 .

Todos os participantes assinaram o Termo de Consentimento Livre e Esclarecido (TCLE), contendo: objetivo do estudo, procedimentos de avaliação, possíveis consequências, procedimentos de emergência, caráter de voluntariedade da participação do sujeito e respeitando os princípios da acessibilidade, confiabilidade, liberdade e responsabilidade. A identidade pessoal de todos os participantes foi mantida em sigilo bem como todos os dados coletados. A participação foi voluntária, foi dada a todos os participantes a liberdade de se retirarem do projeto a qualquer momento sem que houvesse qualquer penalidade.

Todos os voluntários, aleatoriamente, participaram de uma sessão de TC. Os participantes realizaram 16 visitas aos locais destinados ao projeto para a realização de todos os procedimentos: 
Uma sessão de treino concorrente, aleatoriamente. Foi respeitado um intervalo mínimo de 48 horas entre as avaliações físicas e as intervenções. Os participantes foram instruídos a comparecerem em estado hidratado e a continuarem sua dieta normal, abstendo-se do consumo de álcool 24 horas antes das avaliações físicas e das intervenções, que foram realizadas no mesmo período do dia para evitar a influência do ciclo circadiano, com o objetivo de manter a similaridade das condições experimentais.

Aferição da FC foi realizada por meio de um monitor cardíaco marca Polar RS800CX MULTISPORT PACK (Finlândia). Nos momentos pré uma sessão de TC, foram registrados os dados por 10 minutos e considerando para a analise os últimos 5 minutos, para os momentos pós uma sessão de TC foram registrados 5 minutos imediatamente após para análise dos intervalos R-R nos domínios de tempo e frequência da VFC. Os dados do monitor cardíaco foram passados para um computador com capacidade até o Windows 7, por infravermelho e a análise dos dados foi realizada com software de computador Pro Trainer Version 5, seguindo as recomendações da Task Force da European Society of Cardiology e da North American Society of Pacing and Electrophysiology (Malik; Bigger; Camm, 1996);

Sessão de Treinamento Concorrente (TC): Os voluntários realizaram 30 minutos na esteira a 50\% da FCres, e logo em seguida, uma volta no circuito de 9 exercícios (voador peito, remada sentada, leg press $45^{\circ}$, banco flexor, desenvolvimento ombro, tríceps pulley, rosca direta, flexão plantar sentado e abdominal) que envolvia grandes grupos musculares a $40 \%$ de 1RM com 15 repetições cada, o tempo de treino ficou entre 45 e 50 minutos, essas variáveis foram aplicadas com base em alguns estudos relacionados ao tema de trabalho (Borges et al., 2012; Garcia et al., 2014; Santiago et al., 2013; Souza et al., 2011). O TC foi realizado na Ritmus Academia na cidade de Barretos-SP.

Para a análise dos dados foi utilizado o teste de Wilcoxon pareado para comparar se as diferenças médias obtidas pré e pós intervenção são significativas. O nível de significância adotado foi de p<0,05, isto é, $95 \%$ de probabilidade de que estejam certas as afirmativas e/ou negativas denotadas durante as investigações, admitindo-se, portanto, a probabilidade de $5 \%$ para resultados obtidos por acaso.

Para verificar a relação dos resultados, foi utilizado o teste coeficiente de correlação de Spearman, com a classificação de 0,00 a 0,19 como muito fraca; 0,20 a 0,39 como fraca; 0,40 a 0,69 como moderada; 0,70 a 0,89 como forte e 0,90 a 1,00 como muito forte. O tamanho de efeito também foi calculado utilizando o teste de Cohen (d) (1988), os valores de "d" são considerados pequenos se $20 \leq \mathrm{d}<50$; médios se $50 \leq \mathrm{d}<80$ e grandes se $\mathrm{d} \geq 80$.

O presente estudo atendeu as normas para a realização de pesquisa em seres humanos, de acordo com a Resolução 466/2012, do Conselho Nacional de Saúde de 12/12/2012, foi aprovado pelo comitê de ética em pesquisa (CEP) da Universidade Federal do Estado do Rio de Janeiro (UNIRIO) com o CAAE 45756115.5.0000.5285, parecer: 1.180.516. Todos os participantes assinaram o Termo de Consentimento Livre e Esclarecido. Os participantes realizaram 16 visitas aos locais destinados ao projeto para a realização de todos os procedimentos, inclusive uma sessão de TC, aleatoriamente.

\section{Resultados e Discussão}

Foram convidados a participar 611 pessoas que vivem com HIV e que fazem acompanhamento no SAE na cidade de Barretos-SP, desses, 70 pessoas manifestaram interesse em participar. Contudo, participaram do estudo 15 pessoas, 9 mulheres e 6 homens com 50,54 \pm 10,81anos, tempo de uso da TARV de 9,17 $\pm 4,97$ anos e todos apresentando carga viral menor que 40.

Os dados referentes à avaliação antropométrica estão apresentados na Tabela 2, às avaliações de composição corporal para os participantes do sexo masculino mostram que eles estão com uma distribuição de peso normal pelo IMC, com risco moderado de ter problemas cardiovasculares e com uma classificação de muito boa pelo \%G, para as participantes do sexo 
feminino o IMC está no limite do considerado normal para sobrepeso, a RCQ apresenta para elas é classificada como alto o risco de ter algum problema cardiovascular e em relação ao \%G, as participantes pela idade estão numa situação que são consideradas como magras.

Tabela 2. Valores de média \pm Desvio padrão do peso, estatura, IMC, cintura, quadril, RCQ e percentual de gordura de pessoas vivendo com HIV $(\mathrm{n}=15)$.

\begin{tabular}{lll}
\hline Descriçào & Valores & Valores de Referência \\
\hline Peso & $65,19 \pm 11,01 \mathrm{~kg}$ & - \\
Estatura & $1,63 \pm 0,09 \mathrm{~m}$ & - \\
IMC & $24,61 \pm 4,34 \mathrm{~kg} \cdot \mathrm{m}^{-2}$ & $18,60 \mathrm{a} 24,9 \mathrm{~kg} \cdot \mathrm{m}^{-2}$ \\
IMC Masculino & $23,99 \pm 5,22 \mathrm{~kg} \cdot \mathrm{m}^{-2}$ & $18,60 \mathrm{a} 24,9 \mathrm{~kg} \cdot \mathrm{m}^{-2}$ \\
IMC Feminino & $25,03 \pm 3,93 \mathrm{~kg} \cdot \mathrm{m}^{-2}$ & - \\
Cintura & $84,77 \pm 7,93 \mathrm{~cm}$ & - \\
Quadril & $95,57 \pm 6,12 \mathrm{~cm}$ & - \\
RCQ & $0,89 \pm 0,06 \mathrm{~cm}$ & $<0,90 \mathrm{~cm}$ \\
RCQ Masculino & $0,92 \pm 0,04 \mathrm{~cm}$ & $<0,74 \mathrm{~cm}$ \\
RCQ Feminino & $0,87 \pm 0,07 \mathrm{~cm}$ & - \\
$\% G$ & $22,25 \pm 7,37 \%$ & $18,20 \mathrm{a} 21,20 \%$ \\
$\% G$ Masculino & $19,97 \pm 11,23 \%$ & $25,20 \mathrm{a} 28,60 \%$ \\
\%G Feminino & $23,77 \pm 3,13 \%$ & rercentual de gordura corporal;
\end{tabular}

$\mathrm{kg}=$ quilogramas; $\mathrm{m}=$ metros; $\mathrm{kg} \cdot \mathrm{m}^{-2}=$ quilograma por metro quadrado; $\mathrm{cm}=$ centimetros.

Fonte: Autores.

Os resultados das comparações dos momentos pré e pós TC VFC no domínio da frequência foram apresentados no gráfico abaixo. Houve diferença estatisticamente significativa para todos os parâmetros Total Power, LF, HF e LF/HF. 
Gráfico 1. Comparação pré e pós na variabilidade da frequência cardíaca no domínio da frequência com os parâmetros Total Power, Baixa frequência (LF), Alta frequência (HF) em milissegundos ao quadrado (ms2) e a relação LF/HF no Treinamento Concorrente (TC). ${ }^{*} \mathrm{p}=$ diferença estatisticamente significativa em comparação com o momento pré para a mesma intervenção $(\mathrm{p}<0,05)$.
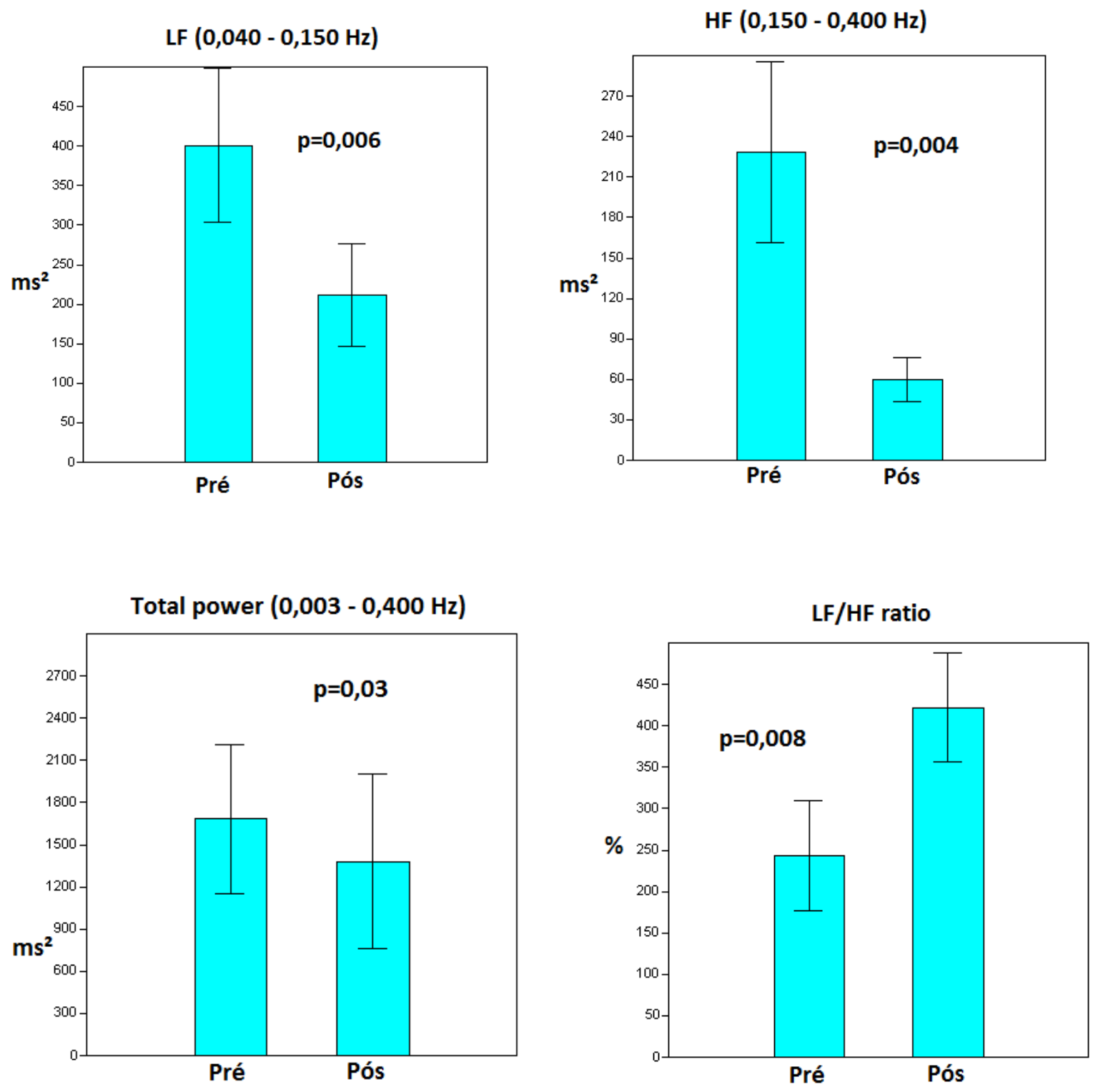

Fonte: Autores.

Os resultados dos gráficos apresentados na figura abaixo mostram as comparações entre os momentos pré e pós na intervenção TC na VFC no domínio do tempo, houve diferença estatisticamente significante para os parâmetros NN, RMSSD, SD1 e pNN50 no TC. 
Gráfico 2. Comparação pré e pós na variabilidade da frequência cardíaca no domínio do tempo com os parâmetros Normalpara-Normal (NN), Desvio padrão dos intervalos NN (SDNN), intervalos de tempo entre ciclos adjacentes como raiz quadrada da somatória do quadrado das diferenças entre os intervalos RR (RMSSD), Descritor padrão 1 (SD1), Descritor padrão 2 (SD2) em milissegundo(ms) e a porcentagem da diferença maior que 50 milissegundos entre intervalos RR adjacentes (pNN50) no Treinamento Concorrente (TC). *p = diferença estatisticamente significativa em comparação com o momento pré para a mesma intervenção $(\mathrm{p}<0,05)$.
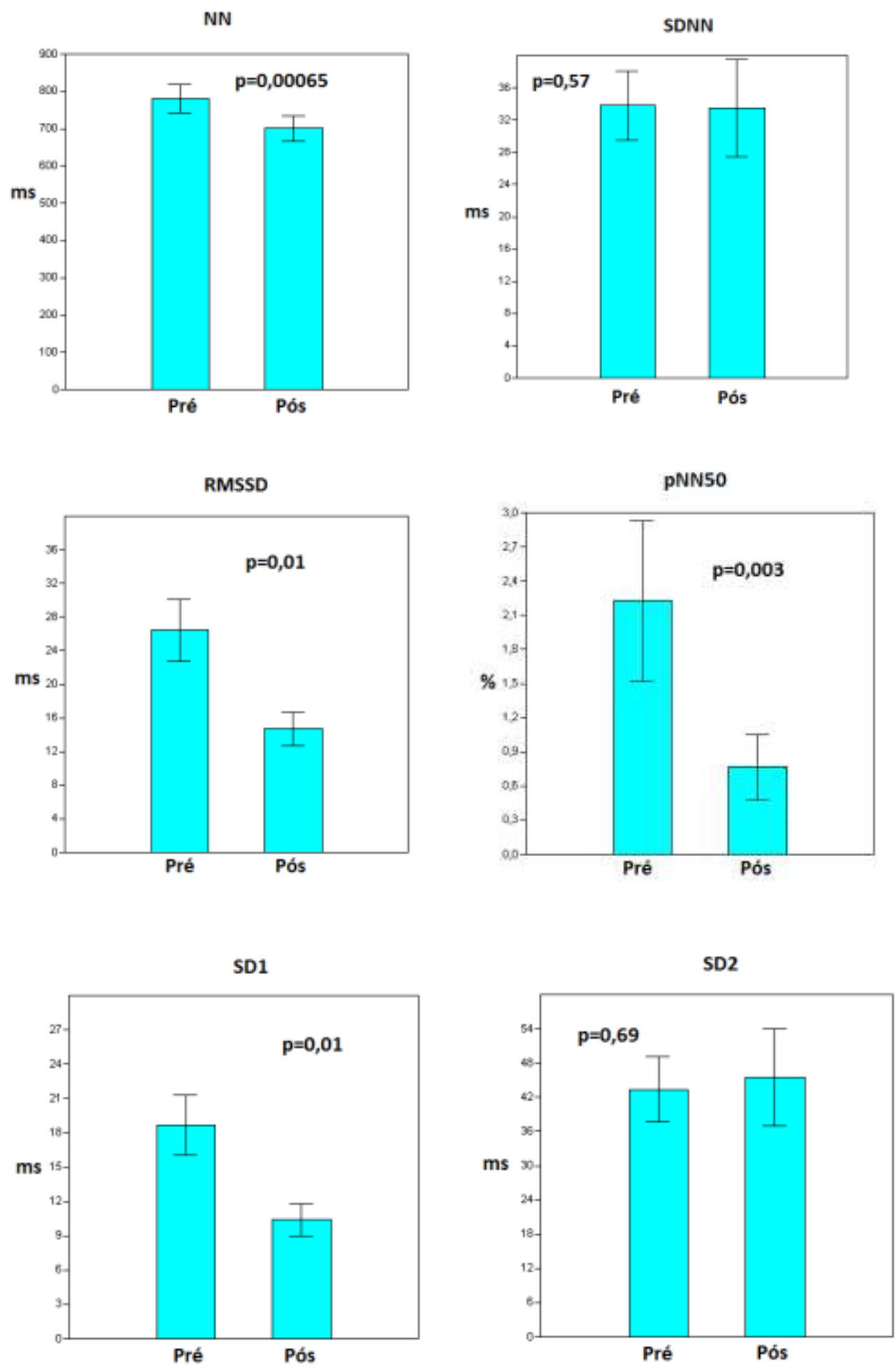

Fonte: Autores. 
Dessa forma foi observado que houve significância na maioria (exceto SDNN e SD2) das análises realizadas, evidenciando alteração da VFC após o TC. Observa-se uma discrepância na análise temporal da ação do simpático/parassimpático em relação as demais variáveis.

As diferenças absolutas e relativas, assim como o tamanho de efeito e suas respectivas classificações das comparações da VFC no domínio do tempo foram apresentadas na tabela 3 abaixo. Houve tamanho de efeito grande nos parâmetros RMSSD $(\Delta \%=79,58 \%$ de redução) e $\operatorname{SD1}(\Delta \%=79,62 \%$ de redução) no $\mathrm{TC}$, o tamanho de efeito médio foi encontrado nos parâmetros $\mathrm{NN}(\Delta \%=11,47 \%$ de redução) e pNN50 $(\Delta \%=190,43 \%$ de redução) no TC, nos outros parâmetros e intervenções não houve efeito.

Tabela 3. Valores de diferença absoluta em ms, do percentual de diferença $(\Delta \%)$, do tamanho de efeito e de sua respectiva classificação na comparação dos momentos pré e pós TC para a variabilidade da frequência cardíaca no domínio do tempo nas variáveis NN, SDNN, RMSSD, SD1, SD2 e pNN50 ( $\mathrm{n}=15)$.

\begin{tabular}{|c|c|c|c|c|c|c|}
\hline & NN & SDNN & RMSSD & SD1 & SD2 & pNN50 \\
\hline $\begin{array}{l}\text { Diferença } \\
\text { absoluta (ms) }\end{array}$ & 80,40 & 0,34 & 11,72 & 8,28 & 2,12 & 1,46 \\
\hline $\begin{array}{l}\text { Percentual de } \\
\text { diferença }(\Delta \%)\end{array}$ & 11,47 & 1,02 & 79,58 & 79,62 & 4,66 & 190,43 \\
\hline $\begin{array}{l}\text { Tamanho de } \\
\text { efeito (TE) }\end{array}$ & 0,58 & 0,02 & 1,02 & 1,02 & 0,08 & 0,70 \\
\hline $\begin{array}{l}\text { Classificação do } \\
\text { tamanho de } \\
\text { efeito }\end{array}$ & Médio & Sem efeito & Grande & Grande & $\begin{array}{l}\text { Sem } \\
\text { efeito }\end{array}$ & Médio \\
\hline
\end{tabular}

$\mathrm{NN}$ = normal-para-normal; SDNN = desvio padrão dos intervalos NN; RMSSD = intervalos de tempo entre ciclos adjacentes como raiz quadrada da somatória do quadrado das diferenças entre os intervalos RR; SD1 = descritor padrão 1; SD2 = descritor padrão 2; pNN50 = porcentagem da diferença maior que 50 milissegundos entre intervalos RR adjacentes; $\mathrm{ms}=$ milissegundos; $\mathrm{TE}=$ tamanho de efeito; $\Delta=$ delta da diferença. Fonte: Autores.

As diferenças absolutas e relativas, assim como o tamanho de efeito e suas respectivas classificações das comparações da VFC no domínio da frequência foram apresentadas na tabela 4. Houve tamanho de efeito grande somente para o parâmetro $\mathrm{HF}(\Delta \%=279,92 \%$ de redução) no TC, houve tamanho de efeito médio nos parâmetros $\mathrm{LF}(\Delta \%=89,04 \%$ de redução) e $\mathrm{LF} / \mathrm{HF}(\Delta \%=42,31 \%$ de aumento) no TC, nos outros parâmetros e intervenções não houve efeito. 
Tabela 4. Valores de diferença absoluta em ms2, do percentual de diferença $(\Delta \%)$, do tamanho de efeito e de sua respectiva classificação na comparação dos momentos pré e pós na CC, TC e AL para a variabilidade da frequência cardíaca no domínio da frequência nas variáveis Total Power, LF, HF e LF/HF (n=15).

\begin{tabular}{|c|c|c|c|c|}
\hline & $\begin{array}{l}\text { Total power } \\
\left(\mathrm{ms}^{2}\right)\end{array}$ & $\begin{array}{l}\mathbf{L F} \\
\left(\mathbf{m s}^{2}\right)\end{array}$ & $\begin{array}{l}\mathbf{H F} \\
\left(\mathbf{m s}^{2}\right)\end{array}$ & $\begin{array}{l}\text { LF/HF } \\
(\%)\end{array}$ \\
\hline $\begin{array}{l}\text { Diferença } \\
\text { absoluta (ms) }\end{array}$ & 302,35 & 188,81 & 168,43 & 178,67 \\
\hline $\begin{array}{l}\text { Percentual de } \\
\text { diferença }(\Delta \%)\end{array}$ & 21,88 & 89,04 & 279,92 & 42,31 \\
\hline $\begin{array}{ll}\text { Tamanho } & \text { de } \\
\text { efeito (TE) } & \end{array}$ & 0,14 & 0,59 & 0,89 & 0,70 \\
\hline $\begin{array}{ll}\text { Classificação } & \text { do } \\
\text { tamanho } & \text { de } \\
\text { efeito } & \end{array}$ & Sem efeito & Médio & Grande & Médio \\
\hline
\end{tabular}

$\mathrm{LF}=$ baixa frequência; $\mathrm{HF}=$ alta frequência; $\mathrm{LF} / \mathrm{HF}=$ relação baixa frequência e alta frequência; $\mathrm{ms}^{2}=$ milissegundos ao quadrado; $\mathrm{TE}=$ tamanho de efeito; $\Delta=$ delta da diferença. Fonte: Autores.

Em um estudo envolvendo soropositivos em uso de TARV durante mais de 44 meses notou-se que eles apresentavam frequência cardíaca aumentada em repouso com diminuição da variabilidade de curto prazo da frequência cardíaca, indicando uma disfunção parassimpática. (Lebech, et al. 2007). Entretanto, um estudo prospectivo realizado em 2012 sugere que a TARV possa não contribuir a curto prazo na função autonômica em indivíduos saudáveis com o vírus HIV. (Chow, et al. 2012).

Encontramos em nosso estudo, após o TC encontramos redução significativa de LF $(\Delta \%=89,04 \%$ e TE $=0,59)$, enquanto $\operatorname{SDNN}(\Delta \%=1,02$ e TE= 0,02) e SD2 $(\Delta \%=4,66$ e TE $=0,08)$ não foram significativos, todos esses parâmetros da VFC indicam, quando há uma redução, uma menor participação simpática. Diante disso, em pessoas que vivem com HIV, que mesmo em repouso a atividade simpática permanece excessiva, quando intervenções (TC) são capazes de reduzi-la, isto pode proporcionar uma melhora do sistema imunológico por modulação das citocinas ou ainda diminui a sobrecarga cardíaca (Elenkov et al., 2000).

Os resultados da VFC no domínio do tempo após TC, na VFC no domínio do tempo diminuiu significativamente nos parâmetros NN, SD1, RMSSD e pNN50, o mesmo ocorreu para o domínio da frequência com o HF, sugerindo que a retomada vagal, que normalmente ocorre após um treinamento físico, não ocorreu ou está retornando lentamente. Uma resposta positiva foi no parâmetro LF, em que houve uma redução significativa, ou seja, uma retirada simpática após o TC, mesmo assim a retirada simpática não foi total, pois o parâmetro $\operatorname{LF} / \mathrm{HF}(\Delta \%=42,31 \%$ de aumento e $\mathrm{TE}=0,70)$, que seu aumento significa atividade simpática, mostrou um aumento significativo após o TC. HF $(\Delta \%=279,92$ e TE $=0,89), \mathrm{LF} / \mathrm{HF}(\Delta \%=42,31$ e TE $=$ $0,70)$

Nossos achados sobre a VFC após o TC, vão ao encontro dos resultados de Borges et al. (2012), os autores compararam a VFC em 13 pessoas vivendo com HIV (43 anos) e 10 soronegativos, mediram a VFC antes, durante e depois de uma corrida na esteira a $60 \%$ do VO2pico. O grupo com HIV diminuiu significativamente os valores de SDNN, pNN50, RMSSD, Total Power, LF e HF em repouso e depois do exercício comparado com o grupo de pessoas soronegativas, semelhante ao nosso estudo, somente com pessoas com HIV. Os autores concluíram que houve reativação vagal mais lenta e retirada simpática, ou seja, o mesmo padrão que encontramos. A justificativa para tal comportamento da VFC em pessoas com HIV se deve à disfunção autonômica apresentada por essa população (Di Masi, 2015), os motivos para serem afetadas são 
vários como, por exemplo, pela neurotoxina causada pelas medicações, principalmente a utilização da estavudina que causa depleção mitocondrial neural e inibição de DNA polymerase (Lebech et al., 2007); Por vasculopatia (Mittal et al., 2004); Pelo efeito neurotrópico do vírus e efeito colateral das drogas antirretrovirais (Wongcharoen et al., 2013).

Durante o exercício há uma predominância simpática, que é retirada após o TC (Borges et al., 2012), como vimos na redução de LF, embora não tenha ocorrido uma retirada completa simpática, como registrado no aumento de LH/HF e também não ocorreu um retorno vagal rápido após o TC, como evidenciado pela diminuição da VFC (NN, SD1, RMSSD, pNN50, Total Power e HF), possivelmente por conta da disfunção autonômica, característica de pessoas que vivem com HIV (Di Masi, 2015).

\section{Conclusão}

Com base nos resultados encontrados e levando em consideração as análises limitadas, e o $\mathrm{n}$ amostral relativamente baixo, podemos tecer as seguintes conclusões para pessoas vivendo com HIV:

A variabilidade da frequência cardíaca dos pacientes em estudo diminuiu após o treinamento concorrente possivelmente pela disfunção autonômica evidenciada na literatura para pessoas vivendo com HIV. Não foi observado aumento da VFC após a intervenção, isso corrobora a hipótese de que pacientes infectados pelo HIV apresentariam alterações cardiovasculares principalmente se somada aos fatores de risco, como obesidade, sedentarismo, hipertensão arterial. Os resultados indicaram hiperatividade do sistema nervoso autônomo simpático ou redução da atividade parassimpática, tanto em repouso quanto após o exercício físico (treinamento concorrente). Tais características podem ter implicações clínicas em pacientes com HIV em HAART, sendo assim há uma necessidade de identificação desses fatores que aumentam o desenvolvimento de complicações cardiovasculares e a promoção de um estilo de vida mais saudável com a associação de medidas farmacológicas e não farmacológicas, como a atividade física e alimentação equilibrada.

Para trabalhos futuros, sugere-se uma investigação aprofundada com um $\mathrm{n}$ amostral maior para fidedignidade dos resultados encontrados e uma análise conjunta com fatores de riscos cardiovasculares e comorbidades para melhor compreender os efeitos da variabilidade da frequência cardíaca em indivíduos HIV positivos em uso de TARV.

\section{Referências}

Artal, F.J.C.(2017). Infectious diseases causing autonomic dysfunction; Clinal Autonomic Research, 28(1), 67-81.

Borges, J.P.; Farinatti, P.T.V. (2011). Regulação autonômica da frequência cardíaca em pacientes infectados pelo HIV . Revista Brasileira de Fisiologia do Exercício - Volume 10 Número 4.

Brasil. (2018) Ministério da Saúde. Boletim Epidemiológico HIV AIDS. Brasília: Ministério da Saúde

Brasil. (2018) Secretaria de Vigilância em Saúde. Boletim Epidemiológico. Ministério da Saúde. Número Especial.

Brasil. (2018). Secretaria de Vigilância em Saúde. Departamento de Vigilância, Prevenção e Controle das Infecções Sexualmente Transmissíveis, do HIV/Aids e das Hepatites Virais. Protocolo Clínico e Diretrizes Terapêuticas para Manejo da Infecção pelo HIV em Adultos / Ministério da Saúde, Secretaria de Vigilância em Saúde, Departamento de Vigilância, Prevenção e Controle das Infecções Sexualmente Transmissíveis, do HIV/Aids e das Hepatites Virais. Brasília : Ministério da Saúde.

Costa, L,A., \& Almeida, A. G. (2015). Patologia cardiovascular associada ao vírus da imunodeficiência humana. Revista Portuguesa de Cardiologia, 34(7-8), $479-491$.

Chaves, A.C.P, et al. (2014) Conhecimentos e atitudes de adolescentes de uma escola pública sobre a transmissão sexual do HIV; Revista Brasileira de Enfermagem. 67(1): 48-53.

Chetty, L., Cobbing, S., \& Chetty, V. (2020). Physical activity and exercise for older people living with HIV: a protocol for a scoping review. Systematic Reviews, 9(1).

Chow, D.C., et al. (2011) Cardiovagal autonomic function in HIV-infected patients with unsupressed HIV viremia. HIV Clinical Trials. 12(3):141-150 
Chow, D., et al. (2012) Effects of antiretro-viral therapy on autonomic function in early HIV infection:A preliminary report. Internacional Journal of Medical Sciences; 9(5), 397-405.

Chow, D.C., et al. (2015) Symptoms of autonomic dysfunction inhuman immunodeficiency virus. Open Forum Infectious Diseases. 2(3), ofv103.

Deeks, S. G., Lewin, S. R., \& Havlir, D. V. (2013). The end of AIDS: HIV infection as a chronic disease. The Lancet, $382(9903)$, $1525-1533$.

Dong, J-G. (2016) The role of heart rate variability in sports physiology. Experimental and therapeutic medicine. v. 11, n. 5, p. 1531-1536,

Esiri, M. M., Morris, C. S., \& Millard, P. R. (1993). Sensory and sympathetic ganglia in HIV-1 infection: Immunocytochemical demonstration of HIV-1 viral antigens, increased MHC class II antigen expression and mild reactive inflammation. Journal of the Neurological Sciences, 114(2), $178-187$.

Fang, X., et al. (2015). Resilience, stress, and life quality in older adults living with HIV/AIDS. Aging \& Mental Health, 19(11), 1015-1021.

Faria, G.; Gude, A.S. \& Lima, A.C.C. (2018) Perfil epidemiológico das notificações de AIDS e infecção pelo HIV na população de Cacoal no período de 2010 a 2016. Revista da Faculdade de Educação e Meio Ambiente -FAEMA, Ariquemes. v.9, n. 2, p.698-705.

Fronteira, I. (2013) Estudos observacionais na era da medicina baseada na evidência, Acta Medica Portuguesa. 26(2):161-170

Gill, J, et al. (2010) Causes of Death in HIV-1 - Infected Patients Treated with Antiretroviral Therapy, 1996-2006: Collaborative Analysis of 13 HIV Cohort Studies. Clinical Infectious Diseases. 50(10), 1387-1396.

Godijk, N.G., et al. Heart Rate Variability, HIV and the Risk of Cardiovascular Diseases in Rural South Africa. Global Heart. $2020 ; 15(1)$ : 17.

Lebech, A-M., et al. (2007) Autonomic dysfunction in HIV patients on antiretroviral therapy: studies of heart rate variability. Clinical physiology and functional imaging, v. 27, n. 6, p. 363-367.

Lima, E.M., et al. Cardiovascular prevention in HIV patients: results from a successful interventionprogram. Atherosclerosis. 204(1), 229-232.

Mccraty, R. \& Shaffer, F. (2015) Heart rate variability: new perspectives on physiological mechanisms, assessment of self-regulatory capacity, and health risk. Global advances in health and medicine, v. 4, n. 1, p. 46-61.

Mcintosh, R. C. (2016) A meta-analysis of HIV and heart rate variability in the era of antiretroviral therapy. Clinical Autonomic Research: Official Journal of the Clinical Autonomic Research Society, v. 26, n. 4, p. 287-294.

Mittal, C.M., et al. (2004) Heart rate variability in human immunodeficiency virus-positive individuals. Internacional Journal of Cardiology. 94(1) :1-6.

Ozemek, C., Erlandson, K. M., \& Jankowski, C. M. (2020). Physical activity and exercise to improve cardiovascular health for adults living with HIV. Progress in Cardiovascular Diseases. 63, 178-183

Pereira, G.F.M., et al. (2019) HIV/aids, hepatites virais e outras IST no Brasil: tendências epidemiológicas. Revista brasileira de epidemiologia. 22(suppl 1): e190001.supl.1

Pinto, T., et al. (2013) Benefícios do exercício físico para pacientes com HIV/AIDS. Revista Hospital Universitário Pedro Ernesto, 12 (4):18-26

Quiles, N., Garber, C., \& Ciccolo, J. (2017). Resting Autonomic Function in Active and Insufficiently Active People Living with HIV. International Journal of Sports Medicine, 39(01), 73-78.

Silva, J., et al. (2018) Estudo Comparativo De Docking Molecular Entre O Inibidor De Protease Saquinavir E O Carotenoide Bixina Como Potencial Inibidor Do Vírus Hiv Tipo I (1Hxb). Revista Expressão Católica Saúde, v. 3, n. 1, p. 35-41.

Tyor, W. R., et al. (1992) Cytokine expression in the brain during the acquired immunodeficiency syndrome. Annals of Neurology, 31(4), 349-360.

Umetani, K., et al. (1998) Twenty-four hour time domain heart rate variability and heart rate: relations to age and gender over nine decades. Journal of the American College of Cardiology, 31(3), 593-601. 